\title{
Comparison of Elbow Joint Angles for Male and Female Bat Swings
}

Elisha Gregory, Rose-Hulman Institute of Technology

Faculty Advisor: Renee Rogge, Rose-Hulman Institute of Technology

\section{Student Paper Abstract}

INTRODUCTION: Hitting has become one of the most studied aspects of the game of baseball. Many studies to date only focus on a specific group of players, like collegiate or major league players, but there have not been any studies comparing the male swing to the female swing. Additionally, most studies look at many different aspects of a swing at distinct times in the swing.

This study concentrates on determining the differences in elbow angle during a bat swing between a male and female hitter. Flexion and extension of the arm muscles control the elbow angle during the swing. Elbow flexion is studied because the bat swing works by first coiling the body and then uncoiling in an efficient way to obtain the highest bat speed at ball impact.1

Comparing the male swing to the female swing will show if the two sexes take a similar action and perform it differently. Clearly there is a difference in performance between the two sexes; males being able to swing faster and hit harder than females. One possibility leading to these differences in outcomes is a difference in elbow angles during the bat swing and ball contact. A difference found in the magnitude of the angle or the angle at which ball contact was made could explain why males have a faster and harder swing speed. Could it be possible that males make ball contact at the optimal elbow angle allowing them to get more power from their swing while females have not yet discovered this optimal angle?

CONCLUSIONS \& RECOMMENDATIONS: As the results show, there is not a significant change in the angle of the upper and lower arm in male baseball swings and female softball swings. Both sexes have similar upper arm flexion throughout the swing especially at time when the bat contacts the ball. The only difference observed was the females swing showed a short period of increased elbow flexion before the upper arm would extend in the swing.

For future research, bat position, leg movement, or angular acceleration of the torso could be compared to see if there is a significant difference between a baseball or softball swing. Also, the resolution used in this study was able to record the entire female softball swing, however because of the quicker bat speed there were gaps in the data of the male baseball swing. Therefore, higher resolution cameras could be used in order to get a better capture the motion of a male baseball swing.

\section{REFERENCES:}

1. Welch, C. M. et al (1995). Hitting a Baseball: A Biomechanical Description, 22 (5), 193201. The Journal of Orthopaedic and Sports Physical Therapy.

ACKNOWLEDGEMENTS: We would like to thank the Rose-Hulman Biomedical Engineering Department, who allowed us to borrow the motion analysis cameras. We also want to thank Dr. Renee Rogge and Dr. Glen Livesay who helped with our research.

\section{Key Words}

Student Paper, Biomedical Engineering, Applied Biology and Biomedical Engineering 


\title{
COMPARISON ELBOW JOINT ANGLES FOR MALE AND FEMALE BAT SWINGS
}

\author{
Elisha Gregory, Samantha Sheets, Brent Witten
}

\author{
Sports Biomechanics Research Group \\ Department of Applied Biology and Biomedical Engineering \\ Rose-Hulman Institute of Technology \\ Terre Haute, IN 47803
}

\section{INTRODUCTION}

Hitting has become one of the most studied aspects of the game of baseball. Many studies to date only focus on a specific group of players, like collegiate or major league players, but there have not been any studies comparing the male swing to the female swing. Additionally, most studies look at many different aspects of a swing at distinct times in the swing.

This study concentrates on determining the differences in elbow angle during a bat swing between a male and female hitter. Flexion and extension of the arm muscles control the elbow angle during the swing. Elbow flexion is studied because the bat swing works by first coiling the body and then uncoiling in an efficient way to obtain the highest bat speed at ball impact. ${ }^{1}$

Comparing the male swing to the female swing will show if the two sexes take a similar action yet perform it differently. Clearly there is a difference in performance between the two sexes; males being able to swing faster and hit harder than females. One possibility leading to these differences in outcomes is a difference in elbow angles during the bat swing and ball contact. A difference found in the magnitude of the angle, or the angle at which ball contact was made, could explain why males have a faster and harder swing speed. Could it be possible that males make ball contact at the optimal elbow angle allowing them to get more power from their swing while females have not yet discovered this optimal angle?

\section{METHODS}

In order to collect data, a motion analysis system was used. Five motion analysis cameras were setup in a sports arena surrounding the right side of a batting tee, which is shown in Figure 1. Light reflective markers were placed on each subject at their wrist, elbow, and shoulder joints. To record the ball being hit, light reflective tape was placed on a baseball and softball as well as the bat.

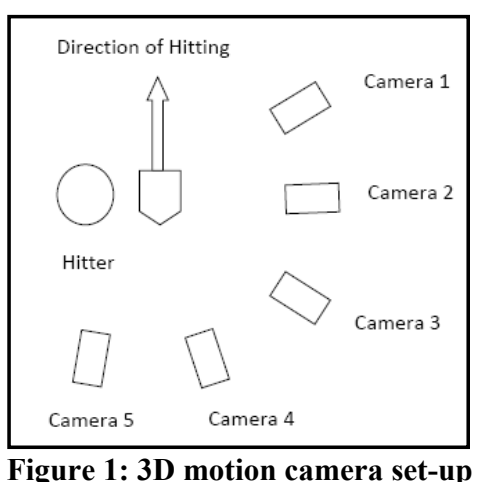

Five softball players and five baseball players were selected from the varsity teams at Rose-Hulman Institute of Technology to hit a ball off a tee. Participants were chosen because they were seen as strong hitters, by their coaches for their respective teams. Five swings were recorded for each player for analysis.

Once the data was recorded, elbow angles throughout the swing were extracted and used to compare the softball players' swings to the baseball players' swings. Also, the elbow angle at bat/ball contact was extracted. Bat/ball contact was determined by finding the frame where the ball began to move from its original position. For each player's five swings, the elbow angles were averaged together as well as the angle at which ball contact was made. The data was shifted to place 
the time point of ball contact was the same for each player allowing for comparison. This information was displayed in graphs: one for softball players and one for baseball players.

\section{RESULTS}

The data collected and analyzed showed some differences and similarities in female and male bat swings. Both females and males showed similar maximum elbow angles with an average females max elbow angle of $167.8^{\circ}$ and an average male maximum elbow angle of $168.3^{\circ}$. Also, females and males starting elbow angles and finishing elbow angles were very similar as shown in Table 1 .

Table 1: Starting and finishing elbow angles (in degrees) for females and males while hitting of a tee.

\begin{tabular}{|c|c|c|}
\hline \multicolumn{2}{|c|}{ females and males while hitting of a tee. } \\
\hline Females & Start & Finish \\
\hline Males & 59 & 116.6 \\
\hline
\end{tabular}

A noticeable but not significant difference between a female and male bat swing is the elbow angle at which ball contact was made. The females' elbow angle at ball contact ranged from $102.2^{\circ}$ to $125.6^{\circ}$ (the gray shaded region in Figure 2), while the males' elbow angle at ball contact ranged from $102.2^{\circ}$ to $119.8^{\circ}$ (the gray shaded region in Figure 3 ). In both females and males there was one hitter with a small elbow angle at ball contact. When these two players' elbow angles were removed, the females' range changed from $121.4^{\circ}$ to $125.6^{\circ}$ and males' range changed from $112.4^{\circ}$ to $119.8^{\circ}$. These new ranges show more of a difference in elbow angle at ball contact between the male and female bat swing. Another noticeable difference is the speed of the swing. This was noticed during data collection and can be seen in the graphs by the increased elbow angle slope in the male hitters compared to the female hitters.

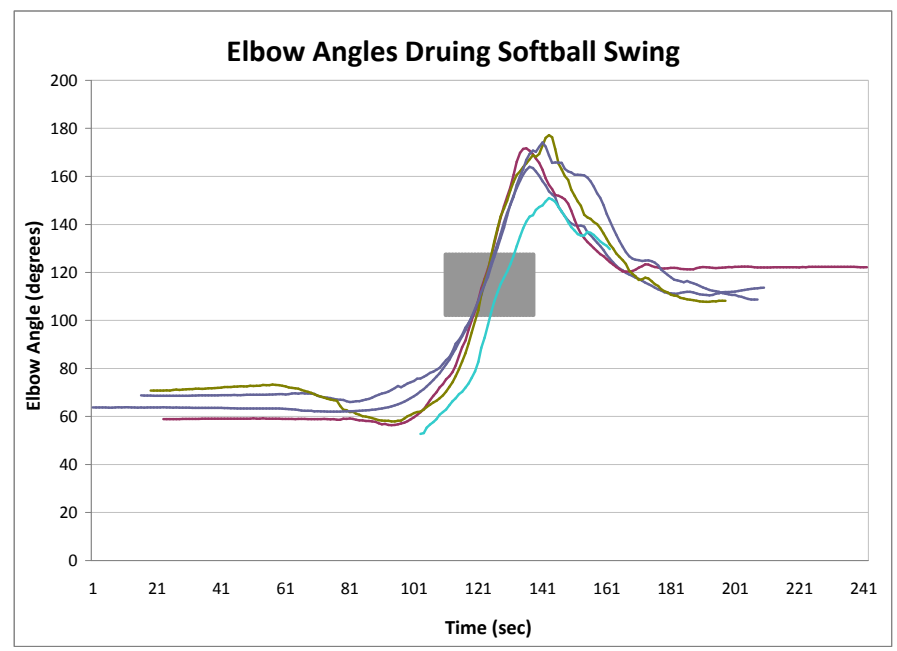

Figure 2: Five female softballball players' average elbow angles while hitting of a tee. The shaded gray area represents the overall range of elbow angles at which ball contact was made for all five players.

\section{DISCUSSION}

This study has shown that there are in fact differences and similarities between the male baseball swing and the female softball swing when focusing on arm angle. After checking the data for normality, a twosample t-test was performed (with $\alpha=0.05$ ) in Minitab in order to determine if there was a true difference in the maximum and minimum elbow angles between the males and females. No statistical difference between the maximum and minimum swings of males and females. Despite this, slight trends can be observed. For example, all of the females tested had a decreasing elbow angle before the angle increased to make ball contact, while this only occurred in two of the male subjects. Also, it was found that males have a much faster swing than females.

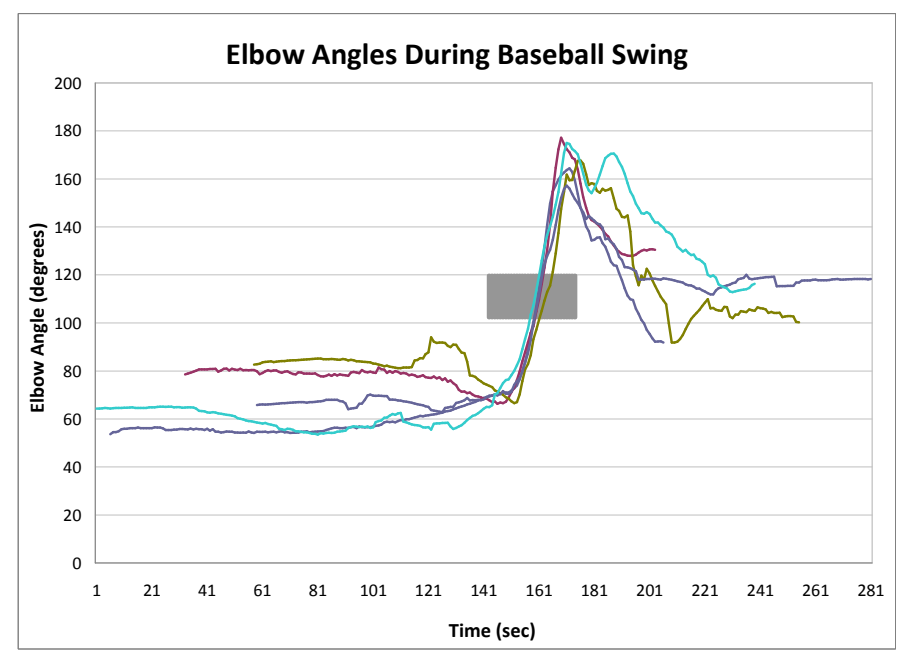

Figure 3: Five male baseball players' average elbow angles while hitting of a tee. The shaded gray area represents the overall range of elbow angles at which ball contact was made for all five players.

\section{CONCLUSIONS \& RECOMMENDATIONS}

As the results show, there is not a significant change in the angle of the upper and lower arm in male baseball swings and female softball swings. Both sexes have similar upper arm flexion throughout the swing especially at time when the bat contacts the ball. The only difference observed was the females swing showed a short period of increased elbow flexion before the upper arm would extend in the swing.

For future research, bat position, leg movement, or angular acceleration of the torso could be compared to see if there is a significant difference between a baseball and a softball swing. Also, the resolution used in this study was able to record the entire female softball swing, however because of the quicker bat speed there were gaps in the data of the male baseball swing. Therefore, higher resolution cameras could be used in order to better capture the motion of a male baseball swing.

\section{REFERENCES}

${ }^{1}$ Welch, C. M. et al (1995). Hitting a Baseball: A Biomechanical Description, 22 (5), 193-201. The Journal of Orthopaedic and Sports Physical Therapy.

\section{ACKNOWLEDGEMENTS}

We would like to thank the Rose-Hulman Biomedical Engineering Department, who allowed us to borrow the motion analysis cameras. We also want to thank Dr. Renee Rogge and Dr. Glen Livesay who helped with our research. 